\title{
FERRITIN LEVELS IN ANCA-ASSOCIATED VASCULITIS: AN ACTIVE MEDIATOR OF THE INFLAMMATORY PROCESS?
}

\author{
Isabele Parente de Brito Antonelli1 ${ }^{1, \star}$, Heitor Furlan Giordano ${ }^{1}$, Bidossessi Wilfried Hounkpe ${ }^{1}$, Samuel Katsuyuki Shinjo ${ }^{1}$ \\ Rosa Maria Rodrigues Pereira ${ }^{1}$
}

1.Universidade de São Paulo, São Paulo (SP), Brazil.

*Corresponding author: isabeleparente08@gmail.com

\section{BACKGROUND}

Ferritin is a protein classically recognized in iron homeostasis and as an acute phase reactant; however, recent advances have shown that it may play a pathogenic role in inflammation. Previous studies have already demonstrated its correlation with disease activity in systemic lupus erythematosus and dermatomyositis. Regarding ANCA-associated Vasculitis (AAV), only one retrospective study found a positive correlation with disease activity, specifically alveolar hemorrhage and renal impairment. The aim of this work is to prospectively assess the association of ferritin with clinical manifestations and disease activity in AAV.

\section{METHODS}

Prospective study between March 2020 and 2021 that included 56 patients with AAV, according to the Chapel Hill Revised Consensus in outpatient follow-up in a Brazilian tertiary center. The exclusion criteria were concomitant autoimmune disease, neoplasms, hepatopathy or pregnancy. The parameters evaluated were demographic data, disease activity by Birmingham vasculitis activity score (BVAS), vasculitis damage index (VDI), current immunosuppression and presence of infection. Chi-squared, Fisher, Wilcoxon signed-rank and Student $t$ tests were used for the univariable analysis.

\section{RESULTS}

Fifty-six patients with AAV were included, 36 patients with granulomatosis with polyangiitis, 11 with eosinophilic granulomatosis with polyangiitis and 9 with microscopic polyangiitis. The mean age was 52.9 years $( \pm 14.5)$, with a predominance of women $(53.57 \%)$ and Caucasians $(73.21 \%)$. The mean VDI was $3.57( \pm 2.13)$ and the mean BVAS was $6.96( \pm 7.48)$. The average value of ferritin was $236.12 \mathrm{ng} / \mathrm{mL}( \pm 414.80)$. Eleven patients had concomitant infection at the time of assessment, with a median BVAS value higher than the group without infection ( 6 vs. $0 ; p=0.01$ ). Notably, there was no significant difference in ferritin in patients with infection compared with ones without infection (median 145.0 vs. 130, $p=0.5$ ). Ferritin was correlated with disease activity by BVAS, with a strong correlation for ocular manifestation.

\section{CONCLUSION}

Ferritin levels increased with disease activity, especially for ocular manifestation. Unlike C-reactive protein, ferritin was not influenced by the presence of infection in AAV patients. This finding highlights the growing importance of ferritin in the assessment of AAV. Not just an "innocent acute phase reagent", but perhaps an active mediator of the inflammatory process and prognostic marker in autoimmune diseases.

\section{KEYWORDS}

Ferritin, ANCA-associated Vasculitis, Vasculitis.

Table 1.

\begin{tabular}{ccc}
\hline BVAS & Correlation Coefficient BVAS-Ferritin & p-value \\
\hline Total & 0.7 & $<0.001$ \\
\hline Eye & 0.85 & $<0.001$ \\
\hline ENT & 0.43 & $<0.001$ \\
\hline Chest & 0.3 & 0.02 \\
\hline Renal & 0.48 & $<0.001$ \\
\hline
\end{tabular}

Realização: 